\title{
Short communication: Intraoperator repeatability and interoperator reproducibility of devices measuring teat dimensions in dairy cows
}

\author{
I. Zwertvaegher, ${ }^{*}$ S. De Vliegher, † J. Baert, ${ }^{*}$ and S. Van Weyenberg*1 \\ ${ }^{*}$ The Institute for Agricultural and Fisheries Research (ILVO), Technology and Food Science Unit, Agricultural Engineering, \\ 9820 Merelbeke, Belgium \\ †M-Team and Mastitis and Milk Quality Research Unit, Department of Reproduction, Obstetrics and Herd Health, Faculty of Veterinary Medicine, \\ Ghent University, 9820 Merelbeke, Belgium
}

\begin{abstract}
Various methods have been applied to measure teat dimensions. However, the accuracy and precision needed to obtain reliable results are often poor or have not yet been investigated. To determine the precision of the ruler, the caliper, and a recently developed 2-dimensional (2D) vision-based measuring device under field conditions, for respectively teat length, teat diameter, and both teat length and diameter, 2 experiments were conducted in which the consistency of measurements within operators (repeatability) and between operators (reproducibility) was tested. In addition, the agreement of the 2D device with the ruler and the caliper was studied. Although the ruler and the 2D device poorly agreed, both methods were precise in measuring teat length when the operators had experience in working with cows. The caliper was repeatable in measuring teat diameter, but was not reproducible. The $2 \mathrm{D}$ device was also repeatable in measuring teat diameter, and reproducible when the operators had experience with the device. The methods had poor agreement, most likely due to the operator-dependent pressure applied by the caliper. Because the 2D device has the advantage of measuring both teat length and teat diameters in a single measurement and is accurate and practical, this method allows efficient and fast collection of data on a large scale for various applications.
\end{abstract}

Key words: measuring device, repeatability, reproducibility, teat dimension

\section{Short Communication}

In dairy cows, the teat represents the interface between the mammary gland and the environment through which milk is drained, and serves as a primary defense mechanism against infections. Consequently,

Received March 7, 2012.

Accepted September 18, 2012. be

${ }^{1}$ Corresponding author: stephanie.vanweyenberg@ilvo.vlaanderen. the teat dimensions and the (mis)match of the teat and the teat cup liner may affect milking characteristics and may influence teat condition and udder health.

Until now, a wide variety of methods to measure teat dimensions have been applied, such as measuring tapes (Tilki et al., 2005), calipers (Tilki et al., 2005), and ultrasonography (Neijenhuis et al., 2001). However, none has been proven to have all of the following characteristics: accuracy (strong agreement between the measured value and the actual value of a variable), precision [i.e., highly repeatable and reproducible (strong agreement between measurements performed by the same operator and performed between operators, respectively)], and practical to use (i.e., limited measuring time and labor).

Recently, we developed a new 2-dimensional (2D) vision-based device for measuring teat dimensions (further referred to as the 2D device; Zwertvaegher et al., 2011). The method uses image analysis, permits fast and objective measurements, and has been shown to be highly accurate (error $<5 \%$ ) during evaluation on artificial teats. Nevertheless, this method has not yet been assessed under field conditions and has not been compared with other available methods.

The aim of this study was to determine the precision of the ruler, the caliper, and the $2 \mathrm{D}$ device, respectively, for measuring teat length, teat diameter, and for both teat length and diameter, when used under field conditions. In addition, the agreement of the results obtained by the $2 \mathrm{D}$ device with those recorded by the ruler and the caliper was assessed.

In experiment 1 , teat length and diameter at the middle of the teat of 8 Holstein-Friesian cows were measured with the 2D device. In addition, teat length and teat diameter were determined using a ruler and a caliper, respectively. Measurements were performed in duplicate and by 2 operators experienced in working with cows, in a tiestall, $2 \mathrm{~h}$ after morning milking (in total, 64 measurements per method per operator). Measurements were performed randomly within each cow (method, operator, and outcome variable teat length and diameter). 
Repeatability was studied within each method for teat length (ruler and 2D device) and for teat diameter (caliper and 2D device) by comparing duplicate measurements, whereas reproducibility of each method was studied by comparing measurements performed by operator A with those performed by operator B. Agreement between the 2D device and the ruler (for teat length) and the caliper (for teat diameter) was calculated by comparing the collected data. Reproducibility and agreement were assessed by comparing only 1 set of data, whereas for repeatability the duplicate measurements were used for obvious reasons.

Prior to statistical analyses, data were checked for normality. First, to determine differences in measurement (repeatability), operator (reproducibility), and measuring method (agreement), paired $t$-tests and Wilcoxon signed-rank tests were performed for teat length and teat diameter, respectively, using SPSS Statistics 19 software (SPSS Inc., 2010). Statistical significance was considered at $P<0.05$. Second, the coefficients of variation were calculated per pair of test results and reported as mean coefficients of variation over all pairs as a measure for precision (repeatability and reproducibility). Furthermore, the overall levels of consistency were reflected by calculating the concordance correlation coefficients (CCC). Bland-Altman plots were produced to visualize the difference between the pairs of test results relative to their mean value with $95 \%$ limits of agreement. Additionally, scatterplots were generated, and regression lines were fit to the data to define the difference relative to the line of perfect agreement (slope $45^{\circ}$ and intercept zero; Arunvipas et al., 2003).

Teats were, on average, $55.79 \mathrm{~mm}$ long and ranged between 35.00 and $90.00 \mathrm{~mm}$. Mean diameter at the middle of the teat was $25.88 \mathrm{~mm}$ and varied between 14.78 and $39.13 \mathrm{~mm}$.

Nonsignificant differences, low coefficient of variation, strong correlations, and mean differences close to zero were present between duplicates and between operators for teat length determined with the ruler (Table 1). The points of the scatterplots clustered around the lines of perfect agreement (Figure 1A).

Duplicates of the diameter at the middle of the teat measured with the caliper showed a nonsignificant difference, low coefficient of variation, strong correlation, and a mean difference close to zero (Table 1). The points of the scatterplot tended to cluster near the line of perfect agreement (Figure 1B). Measurements significantly differed between operators, coefficient of variation was high, correlation was poor, and mean difference in measurements performed by the 2 operators was $2.51 \mathrm{~mm}$ (Table 1). The linear regression line fitted to the scatterplot clearly differed from the line of perfect agreement (Figure 1B).

Table 1. Levels of statistical significance, coefficients of variation, concordance correlation coefficients (CCC), and mean difference and 95\% lower and upper limits of agreement of the Bland-Altman plots for teat length measured with the ruler and the 2-dimensional (2D) device, and for diameter at the middle of the teat measured with the caliper and the $2 \mathrm{D}$ device

\begin{tabular}{|c|c|c|c|c|}
\hline \multirow[b]{2}{*}{ Item } & \multicolumn{2}{|c|}{ Teat length } & \multicolumn{2}{|c|}{ Diameter at the middle of the teat } \\
\hline & Ruler & $2 \mathrm{D}$ device & Caliper & $2 \mathrm{D}$ device \\
\hline $\mathrm{CV}^{2}$ & 2.94 & 4.43 & 3.31 & 1.56 \\
\hline $\mathrm{CCC}^{3}$ & 0.95 & 0.92 & 0.93 & 0.97 \\
\hline Mean difference (limits of agreement) ${ }^{4}$ & $-0.03(-7.31 ; 7.25)$ & $-0.85(-8.97 ; 7.26)$ & $-0.23(-3.17 ; 2.71)$ & $-0.13(-1.75 ; 1.48)$ \\
\hline $\mathrm{CV}^{6}$ & 2.86 & 4.19 & 8.37 & 1.89 \\
\hline $\mathrm{CCC}^{7}$ & 0.96 & 0.94 & 0.65 & 0.95 \\
\hline Mean difference (limits of agreement) ${ }^{8}$ & $-0.66(-7.40 ; 6.09)$ & $0.36(-7.36 ; 8.07)$ & $-2.51(-7.16 ; 2.14)$ & $0.26(-1.77 ; 2.29)$ \\
\hline \multicolumn{5}{|l|}{ Agreement } \\
\hline Significance level ${ }^{9}$ & \multicolumn{2}{|c|}{$<0.001$} & \multicolumn{2}{|c|}{$<0.001$} \\
\hline $\mathrm{CCC}^{10}$ & \multicolumn{2}{|c|}{0.86} & \multicolumn{2}{|c|}{0.50} \\
\hline
\end{tabular}

${ }^{1}$ Level of significance of the paired $t$-test (teat length) and the Wilcoxon signed rank test (teat diameter) between duplicates within each method.

${ }^{2}$ Coefficient of variation between duplicates within each method (\%).

${ }^{3}$ Concordance correlation coefficient between duplicates within each method.

${ }^{4}$ Mean difference (and 95\% lower and upper limit of agreement) of the Bland-Altman plots between duplicates within each method (mm).

${ }^{5}$ Level of significance of the paired $t$-test (teat length) and the Wilcoxon signed rank test (teat diameter) between operators within each method.

${ }^{6}$ Coefficient of variation between operators within each method (\%).

${ }^{7}$ Concordance correlation coefficient between operators within each method.

${ }^{8}$ Mean difference (and 95\% lower and upper limit of agreement) of the Bland-Altman plots between operators within each method (mm).

${ }^{9}$ Level of significance of the paired $t$-test (teat length) and the Wilcoxon signed rank test (teat diameter) between methods.

${ }^{10}$ Concordance correlation coefficient between methods. 
A

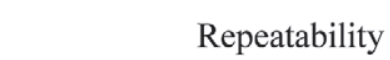

Ruler
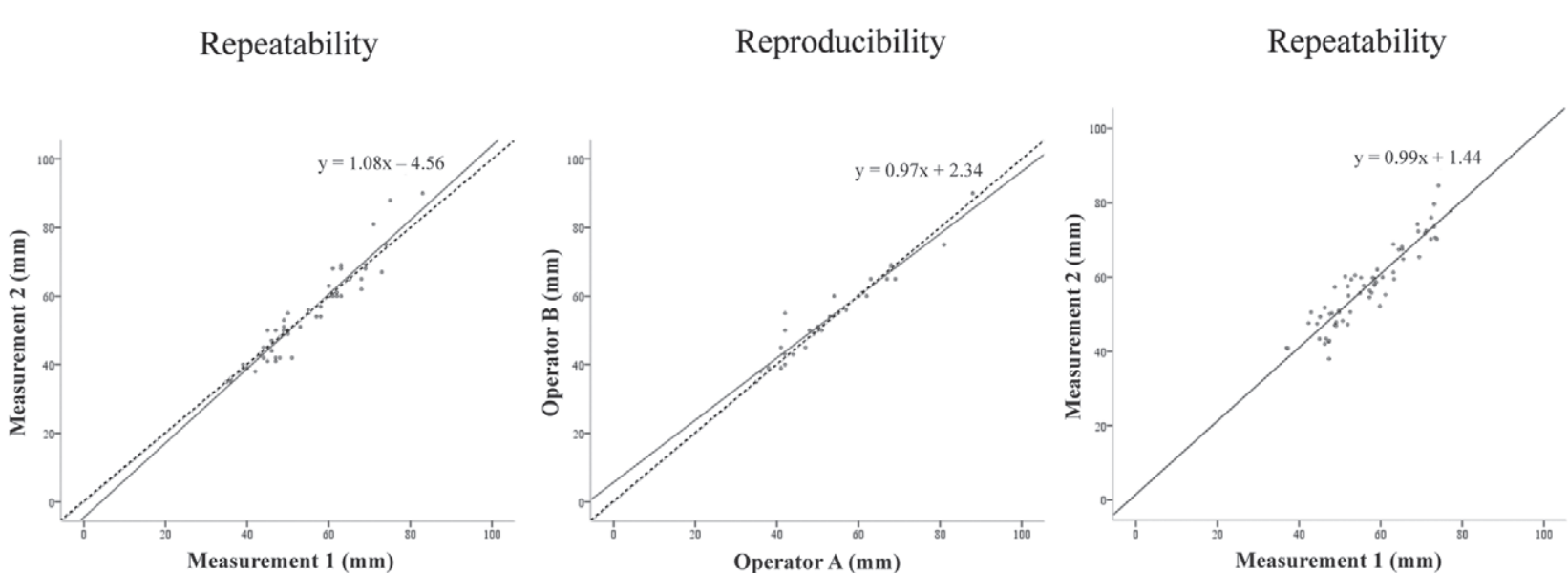

2D device

Reproducibility

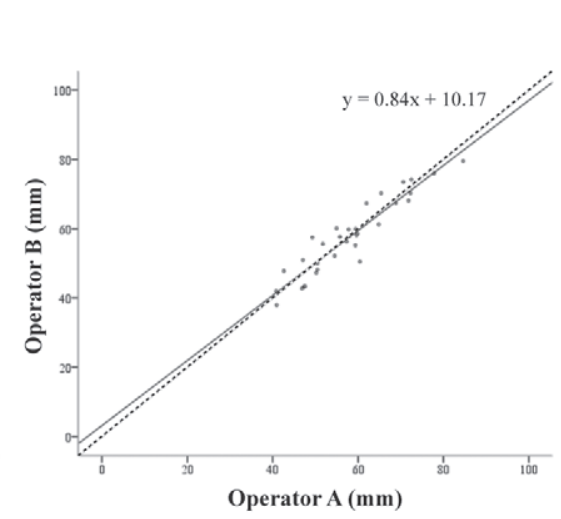

Reproducibility

B

\section{Caliper}

Repeatability

Reproducibility

\section{D device}

Repeatability

Reproducibility
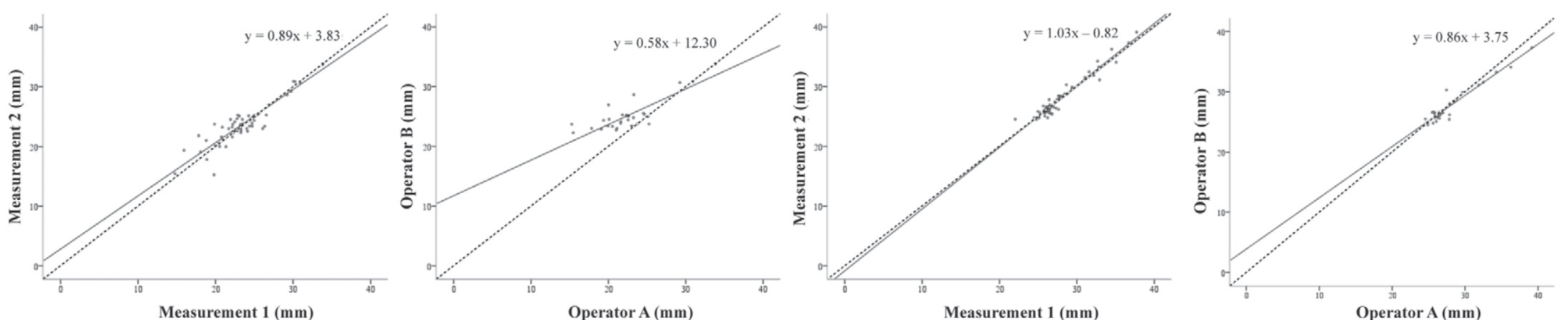

Figure 1. Scatterplots (dotted = line of perfect agreement; solid = linear regression line fit to the data) for repeatability and reproducibility of teat length measured with the ruler and the 2-dimensional (2D) device (A) and of diameter at the middle of the teat measured with the caliper and the $2 \mathrm{D}$ device (B). 
Nonsignificant differences, low coefficient of variation, strong correlations, and mean differences close to zero were found between duplicates and between operators for both teat length and teat diameter measured with the 2D device (Table 1). The linear regression lines fitted to the data approached the line of perfect agreement (Figure 1A and 1B).

The mean teat length measured with the $2 \mathrm{D}$ device was significantly longer (mean $\pm \mathrm{SD}$ ) than that obtained by the ruler $(57.79 \pm 10.89 \mathrm{~mm}$ vs. $54.23 \pm$ $12.37 \mathrm{~mm}$; Table 1). Although CCC was 0.86 , the regression line differed from the line of perfect agreement when the values of the $2 \mathrm{D}$ device were plotted against those of the ruler (Figure 2A). The mean difference between the 2 methods was $3.56 \mathrm{~mm}$. The upper and lower limits of agreement were, respectively, $13.61 \mathrm{~mm}$ and $-6.49 \mathrm{~mm}$.

The average diameter (mean $\pm \mathrm{SD}$ ) at the middle of the teat measured with the 2D device was significantly broader than that measured with the caliper (27.82 \pm $3.24 \mathrm{~mm}$ vs. $24.11 \pm 3.94 \mathrm{~mm}$ ). Correlation was poor (Table 1) and the scatterplot shows a clear difference between the points and the line of perfect agreement (Figure 2B). The mean difference was $3.71 \mathrm{~mm}$ and the $95 \%$ limits of agreement were 8.50 and $-1.07 \mathrm{~mm}$.

An additional experiment was conducted to investigate the precision (repeatability and reproducibility) of the $2 \mathrm{D}$ device in practice more extensively. Teat length and diameter at the middle of the teat were measured with the $2 \mathrm{D}$ device. For studying repeatability, the teats

A

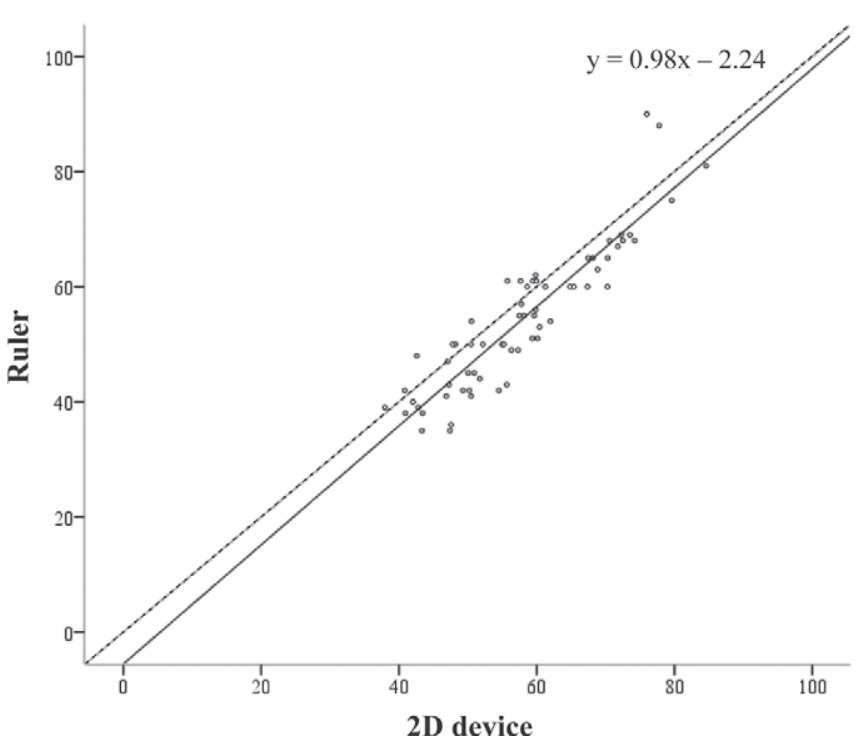

of 19 Holstein-Friesian cows were measured 5 times by 1 operator, with measurements being performed in a tiestall, $3 \mathrm{~h}$ before evening milking. Reproducibility was assessed by measuring the teats of 8 Holstein-Friesian cows once by 8 different operators, with measurements being done $3 \mathrm{~h}$ after morning milking in a tandem milking parlor. Five of the operators were familiar with working with cows (cow experience) and 3 of those 5 were also experienced in working with the $2 \mathrm{D}$ device (tool experience).

Prior to statistical analysis, data were checked for normality. First, to determine whether teat length was associated with measurement (repeatability) or operator, cow experience, or tool experience of the operators (reproducibility), repeated-measures ANOVA were performed, whereas for teat diameter, nonparametric Friedman tests were used (SPSS Statistics 19). Statistical significance was considered when $P<0.05$. Second, coefficients of variation were calculated for all measurements within operator (repeatability), for all operators in general, and for all operators with cow or tool experience (reproducibility), respectively. In addition, CCC, scatterplots and Bland-Altman plots were generated for teat length and teat diameter. For repeatability, the first measurement was compared with the last measurement. For reproducibility, the effect of cow and tool experience on teat length and diameter was examined and visualized for 2 randomly selected persons with cow experience (teat length) and tool experience (teat diameter).

Figure 2. Scatterplots (dotted = line of perfect agreement; solid = linear regression line fit to the data) for agreement of teat length measured with the ruler and the 2-dimensional (2D) device (A) and of diameter at the middle of the teat measured with the caliper and the 2D device (B). 
In the assessment of repeatability, teat length was, on average, $55.13 \mathrm{~mm}$ and ranged between 34.29 and $87.23 \mathrm{~mm}$. No significant difference was found with the repeated-measures ANOVA between teat length measurements performed by the same operator $(P>0.05)$. Coefficient of variation over all measurements was low $(3.26 \%)$. The scatterplot shows a strong correlation between measurement 1 and $5(\mathrm{CCC}=0.92)$ and the mean difference was $0.07 \mathrm{~mm}$, with upper and lower limits of agreement ranging between 6.94 and -6.81 $\mathrm{mm}$.

The mean diameter at the middle of the teat was $26.88 \mathrm{~mm}$ and teats varied between 21.18 and 36.79 $\mathrm{mm}$. The Friedman test indicated that measurement had no significant effect on the diameter at the teat middle $(P>0.05)$. The coefficient of variation of all diameter measurements was low $(1.74 \%)$. Measurement 1 and 5 were strongly correlated $(\mathrm{CCC}=0.94)$ and the mean difference between the 2 measurements was $-0.09 \mathrm{~mm}$. The upper and lower limits of agreement ranged between 1.83 and $-2.01 \mathrm{~mm}$.

In the assessment of reproducibility, teats were, on average, $54.77 \mathrm{~mm}$ long and ranged between 32.16 and $94.67 \mathrm{~mm}$. The repeated-measures ANOVA showed that operator significantly affected teat length measurements $(P<0.001)$, whereas no significant differences were present within the group of operators with cow experience $(P>0.05)$ or within the group of operators with tool experience $(P>0.05)$. The coefficients of variation were $7 \%$ for operators in general, $6.02 \%$ for operators with cow experience, and $5.27 \%$ for toolexperienced operators. A strong correlation $(\mathrm{CCC}=$ 0.93) was found between the teat length measurements of the 2 randomly selected operators with cow experience. Measurements differed, on average, $1.43 \mathrm{~mm}$. The 95\% upper and lower limits of agreement were 10.85 and $-7.98 \mathrm{~mm}$, respectively.

The mean diameter at the middle of the teat was $25.07 \mathrm{~mm}$ and ranged between 19.82 and $34.48 \mathrm{~mm}$. The Friedman test indicated that diameter measurements at the middle of the teat significantly differed between operators $(P<0.001)$ and between operators with cow experience $(P=0.006)$, but operators with tool experience did not significantly differ $(P>0.05)$. The coefficients of variation were $2.3,2.16$, and $1.88 \%$ for operators in general and for operators with cow and tool experience, respectively. The diameter measurements performed by the 2 randomly selected operators with tool experience were strongly correlated $(\mathrm{CCC}=$ $0.97)$. The mean difference between the 2 operators was $0.25 \mathrm{~mm}$ and the $95 \%$ limits of agreement of the BlandAltman plots were situated at 1.91 and $-1.42 \mathrm{~mm}$.

The results of both experiments indicate that the ruler was both repeatable and reproducible in measur- ing teat length when the operators had experience in working with cows. As operators in field conditions are assumed to have cow experience, the effect of cow experience was not examined. Similar, the caliper was highly repeatable in measuring diameter at the middle of the teat but was shown not to be reproducible. We hypothesize that the pressure applied by the caliper on the teat during measurements will influence the results. A low coefficient of variation was found between duplicates performed by the same operator (ca. 3\%), whereas a much higher coefficient of variation was present between operators (ca. 8\%). This high coefficient of variation suggests that the applied pressure is operator dependent. To obtain precise results with the caliper, measurements should, therefore, be performed by 1 operator or operators should measure at equal pressure. In a previous study by Hamann et al. (1988), a coefficient of variation of 8 to $10 \%$ was reported for teat-end thickness measurements performed with the modified cutimeter by the same operator. A higher degree of precision was obtained with the electronic caliper device that uses a pressure sensor that ensures that identical pressure is applied to the teat (Hamann et al., 1988).

The $2 \mathrm{D}$ device was repeatable in measuring teat length, although experience in working with cows is needed to obtain reproducible results. Furthermore, the method was shown to be repeatable and, when the operators had experience with the tool, reproducible in measuring diameters of the teat. The effect of cow and tool experience on repeatability was not examined; nevertheless, some training in working in close interaction with cows and with the measuring device is advised.

The 2D device measured, on average, significantly longer teats than the ruler. This finding can be explained by the fact that the point at which the udder ends and the teat begins is not anatomically discernible at the exterior of the teat, potentially hindering teat measurements. A part of the udder might be inserted through the opening of the $2 \mathrm{D}$ device during measurements and, consequently, be included in the teat-length values. Equally, the ruler, but also other measuring methods, might experience problems with distinguishing the teat from the udder. Nonetheless, the 2 methods were found to be repeatable and reproducible when operators were familiar with cows, and they had similar precision in measuring teat length.

Poor agreement was found between the 2D device and the caliper. The 2D device consistently measured broader teats than the caliper. Smaller diameters measured with the caliper were probably due to the pressure applied by operators, inherent to the instrument.

Although the studied teat measuring devices poorly agreed in teat length and teat diameter measurements, this is trivial when comparing measurements performed 
with the same device, and for the caliper by the same operator. Therefore, all 3 methods allow to be used for various purposes. First, the devices can be used in practice as advisory tools for consulting organizations to help farmers select the most suitable liner for their herd or to select toward more uniform teat dimensions. Second, the methods can be applied in research to investigate the relationship between teat dimensions and teat condition or udder health.

In conclusion, the ruler and the $2 \mathrm{D}$ device were both precise in measuring teat length when the operators had experience with cows. The methods, however, had poor agreement. The 2D device measured, on average, longer teats than the ruler, most likely due to a part of the udder being inserted in the device during measurements. The $2 \mathrm{D}$ device was also repeatable in measuring teat diameter and reproducible when the operators had experience with the device. The caliper was repeatable in measuring teat diameter, but was not reproducible. The methods poorly agreed, with smaller teats measured by the caliper probably because of the operatordependent pressure applied by this device.

\section{ACKNOWLEDGMENTS}

This research was funded by the Institute for the Promotion of Innovation through Science and Technol- ogy in Flanders (IWT-Vlaanderen, Brussels, Belgium; grant no. 50670), the Institute for Agricultural and Fisheries Research (ILVO, Merelbeke, Belgium; PhD scholarship), and Ghent University (UGent, Ghent, Belgium). The authors thank Morgan Carlens for his contribution during his bachelor thesis work at ILVO, and the panel of operators, the technical staff of ILVO, and the Animal Sciences Unit of ILVO for the field work support.

\section{REFERENCES}

Arunvipas, P., J. A. VanLeeuwen, I. R. Dohoo, and G. P. Keefe. 2003. Evaluation of the reliability and repeatability of automated milk urea nitrogen testing. Can. J. Vet. Res. 67:60-63.

Hamann, J., B. Nipp, and G. A. Mein. 1988. Improving milk quality by improving teat condition: A new method for measuring teat tissue-reactions to milking. Milchwissenschaft 43:651-653.

Neijenhuis, F., G. H. Klungel, and H. Hogeveen. 2001. Recovery of cow teats after milking as determined by ultrasonographic scanning. J. Dairy Sci. 84:2599-2606.

SPSS Inc. 2010. IBM SPSS Statistics 19 Core System User's Guide. IBM Corp., New York, NY.

Tilki, M., Ş. İnal, M. Çolak, and M. Garip. 2005. Relationships between milk yield and udder measurements in Brown Swiss cows. Turk. J. Vet. Anim. Sci. 29:75-81.

Zwertvaegher, I., J. Baert, J. Vangeyte, A. Genbrugge, and S. Van Weyenberg. 2011. Objective measuring technique for teat dimensions of dairy cows. Biosystems Eng. 110:206-212. http://dx.doi. org/10.1016/j.biosystemseng.2011.09.009. 